\title{
Preferred drug lists: Potential impact on healthcare economics
}

\section{Kimberly Ovsag \\ Sabrina Hydery \\ Shaker A Mousa}

Pharmaceutical Research Institute at Albany College of Pharmacy, Albany, New York, USA
Correspondence: Shaker A Mousa Pharmaceutical Research Institute at Albany College of Pharmacy, $106 \mathrm{New}$ Scotland Avenue, Albany, NY 12208, USA

Tel +I 5186947397

Fax+I 5186947392

Email mousas@acp.edu
Objectives: To analyze the implementation of Medicaid preferred drug lists (PDLs) in a number of states and determine its impact on quality of care and cost relative to other segments of healthcare.

Methods: We reviewed research and case studies found by searching library databases, primarily MEDLINE and EBSCOHost, and searching pertinent journals. Keywords initially included "drug lists," "prior authorization," "prior approval," and "Medicaid." We added terms such as "influence use of other healthcare services," "quality of care," and "overall economic impact." We mainly used primary sources.

Results: Based on our literature review, we determined that there are a number of issues regarding Medicaid PDLs that need to be addressed. Some issues include: (a) the potential for PDLs to influence the utilization of other healthcare services, (b) criteria used by Medicaid for determining acceptance of drugs onto a PDL, (c) the effect of PDL implementation on compliance to new regimens, (d) the potential effects of restricting medication availability on quality of care, (e) administrative costs associated with PDLs, and (f) satisfaction rates among patients and medical providers. This review highlighted expected short-term cost savings with limited degree of compromised quality of PDL implementation, but raised the concern about the potential long-term decline in quality of care and overall economic impact.

Conclusions: The number of concerns raised indicates that further studies are warranted regarding both short-term cost benefits as well as potential long-term effects of Medicaid PDL implementation. Objective analysis of these effects is necessary to ensure cost-effectiveness and quality of care.

Keywords: preferred drug lists, medicaid, healthcare costs, managed care

Over the past decades, the costs associated with healthcare in the United States have steadily increased. In 2002, the Center for Medicaid and Medicare Services (CMS) found that the US spends nearly $\$ 1.6$ trillion toward healthcare, which was almost $15 \%$ of the gross domestic product (Mattera 2004). In response to the ever-increasing costs associated with healthcare, many policies were initiated through the years; one such policy at the state level is utilization of a preferred drug list (PDL) as part of the Medicaid Preferred Drug Program (PDP). A PDL is a list of medications that Medicaid will cover the cost for without the need to request a prior authorization (PA). PDLs are comprised of medications that either are generic formulations or are the result of price negotiations between the pharmaceutical companies and Medicaid. Because of the cost savings, physicians in most states are required to either prescribe medications on the PDL or receive prior authorization to do otherwise (Gencarelli 2003).

To date, approximately 33 states in the nation have implemented the use of PDLs as a part of their Medicaid programs (Silow-Carroll and Altera 2004). In New York State, this policy has been proposed continuously since 2003-2004 under the expectation that it would curb runaway Medicaid costs, thereby saving taxpayers and the government 
money. However, many physicians, pharmaceutical companies, patients, advocates, senators, and assembly members opposed the bill (Martinez 2003; Seay 2004). Their concerns focused on the possibility that, in the pursuit of short-term decreases in costs by limiting drugs offered to patients, Medicaid might actually be increasing the long-term costs that would accrue; costs due to increased hospitalization because of ineffective or suboptimal therapy and the increased flow of paperwork due to authorization requests. Thus, there would be decreased quality of care further down the line.

The healthcare budget crisis, which is pervasive throughout the country, has prompted many states to look for ways to decrease prescription drug spending. One method used by Medicaid for cost-cutting purposes is required rebates or discounts. According to federal law, pharmaceutical companies must provide prescription drugs to Medicaid at a discounted price (Hamel and Epstein 2004). Many states have also pooled their resources and collective influence together to achieve supplemental rebates beyond the federal requirement from a number of drug companies. Some propositions for cutting Medicaid costs have come from taking a cue from managed care organizations (MCOs). In addition to such rebates, Medicaid can also keep costs low by cost sharing. This concept offers a tiered co-payment system so that beneficiaries pay a certain fee for their prescription drugs, depending on the actual cost of the drugs, thus transferring some of the responsibility for cost of prescription drugs to the patient. However, such a system is much more feasible and much more widely used among private insurers than Medicaid. Medicaid beneficiaries are much less able to make high co-payments (Hamel and Epstein 2004). In addition, a study done with a private insurance provider that switched to a tiered co-payment system indicated that such a change led to significant discontinuity of treatment, which could jeopardize quality of care (Huskamp et al 2003). If such results are seen among patients who have the financial ability to pay higher co-payments, then one could reasonably expect more of the same for patients in the Medicaid community, where such financial abilities are hindered (Gleason et al 2005). For example, three to five different medications might be required for effective management of hypertension. Increasing the patient co-payment would compromise the quality of care in certain cases attributed to the issue of affordability.

Use of drug restrictions or PDLs is another attempt at cost-cutting techniques within healthcare. Drug restrictions are given various names, depending on how strict they are and who enforces them (ie, Medicaid or MCOs). For example, many MCOs use formularies to control drug costs.
A formulary is a list of medications that an MCO deems to be most medically appropriate and cost-effective. If a formulary is open, the physicians make the ultimate decision about use of formulary or non-formulary drugs. On the other hand, a closed formulary does not give such leeway (Carroll 2002). If physicians wish to prescribe a non-formulary medication in a closed formulary, they must go through a prior approval or prior authorization (PA) process in which they must show a special need for the non-formulary medication. MCOs use these methods to control drug costs by decreasing utilization of costly drugs that can be substituted by inexpensive drugs.

Medicaid has adopted PDLs, an idea similar to formularies, which have been implemented in many states, including Michigan, Illinois, Louisiana, North Carolina, Maine, Florida, and Oregon. The PDL places certain prescription drugs on a list, thereby limiting the drugs that physicians can prescribe to their Medicaid patients to the "preferred" drugs on the PDL. Although the PDL may vary from state to state, most PDLs include generic substitutes and less costly innovative medications within the same class as more expensive ones. Just as with MCOs, physicians can request a PA in specific cases in which they believe a patient needs an unlisted drug (Curtiss 2003). The idea of the PDL is two-fold: first, it reduces costs by persuading physicians to switch, and pharmacists to recommend, prescription drugs to equivalent, less expensive drugs that are usually preferred by MCOs (Carroll 2002); second, it gives Medicaid the leverage it needs to negotiate lower prices, above and beyond rebates required federally, with pharmaceutical companies that want to be included in the PDL (Hamel and Epstein 2004).

\section{Factors that influence healthcare costs}

At face value, such programs seem like a great way to reduce healthcare costs, via reduction of drug spending. Nevertheless, there are other issues aside from immediate cost reductions that must be considered. When a change must be made in a beneficiary's prescription due to implementation of a PDL, there may be other unintended effects such as the utilization of other healthcare services, patient compliance with the new regimen, quality of care, physician and patient satisfaction, and associated administrative costs. In this report, we will review research studies that examined the impact of PDL implementation on healthcare costs as well as quality of care. By searching research databases available through the Albany College of Pharmacy (ACP) library system, we were able to identify relevant journal articles concerning the topic 
at hand. The two databases searched included MEDLINE and EBSCOHost. In addition, specific journals including The New England Journal of Medicine, American Journal of Managed Care, and Journal of Managed Care Pharmacy, were specifically searched for publications relating to our research question via the journal search tool available through the ACP library system. Initially keywords such as "drug lists", "prior authorization", "prior approval", and "Medicaid" were used to perform our literature search. However, once specific factors were established such as utilization of other healthcare services, differences in restrictiveness of formularies, and overall economic impact, these terms were used to run the literature search.

Once articles were collected through the database, they were examined to make certain that they were primary sources indicating the effects of PDL implementation. If they were secondary sources, the primary sources were identified through the works-cited sections of the secondary sources; these primary sources were sought out, reviewed, and included in our study. After all relevant research was gathered, the information was combined to make some conclusions as to the effects of PDL implementation on healthcare costs and quality of care.

\section{Effect on utilization of other health care services}

The most common and most studied concern regarding PDLs is the idea that medication restrictions may tend to increase the utilization of other healthcare services, such as hospital and physician visits. The economic burden of such an effect is illustrated in Figure 1, which shows the economic impact of coronary artery disease. Fifty percent of the cost comes from direct management of the disease, and the other Fifty percent from indirect costs, including productivity loss, morbidity, and mortality. Medications are one of the less expensive segments that are part of the direct cost of the disease. Decreased quality of care can lead to an increase in hospital visits, thus increasing both direct and indirect costs through loss of productivity. The use of more efficacious medication would in turn reduce the number of hospital visits thus curtailing one of the most expensive segments of the healthcare dollar.

\section{Coronary Heart Disease Economic Impacts in the US}

\section{Estimated Total Cost $=\$ 118.2$ Billion}

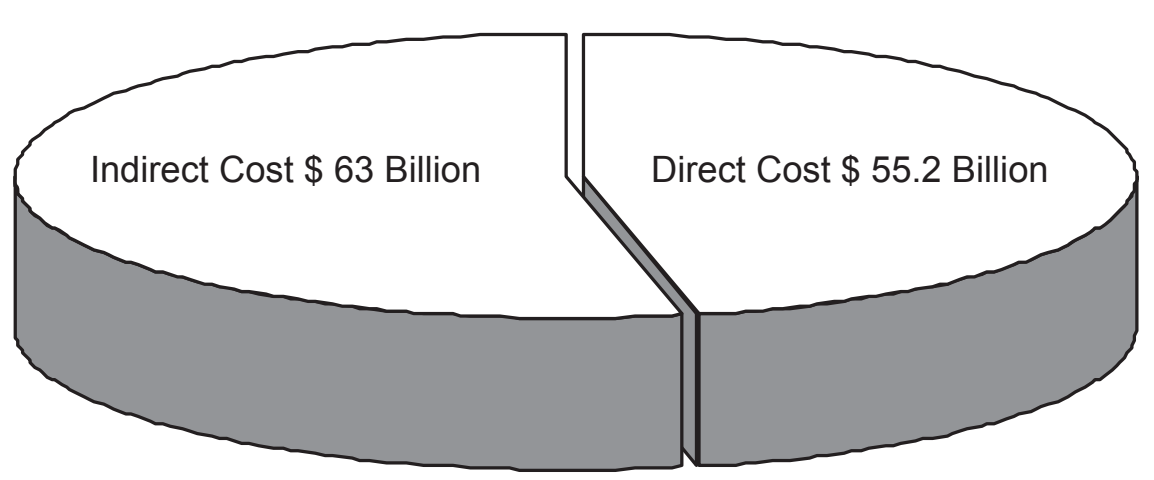

Direct Cost:

-Hospital/nursing home

-Physicians/Other professionals

-Medication

-Home health/other medical durables

\section{Indirect Cost:}

-Loss of productivity due to morbidity or mortality

Figure I The economic impact of coronary heart disease in the United States (AHA 2000). 
In one study, it was found that Medicaid patients with cardiovascular conditions who were restricted by PDLs faced a statistically significant increase in number of outpatient hospital visits six months after PDL implementation, in comparison to non-Medicaid patients with cardiovascular conditions who were not restricted by PDLs. In fact, Medicaid reimbursement among cardiovascular patients in the state increased during the year of the study. It was stated in the study that hospital or physician visits were taken into account if the primary reason for the visit was cardiovascular in nature (Murawski and Abdelgawad 2005). The cost analysis performed in this study did show more modest results than anticipated due to the small sample size and difficulty in accounting for certain variables. The differences-in-differences approach used, however, worked to reduce or eliminate bias. The trends seen in this study show an importance in the need for more attention to the long-term financial impact of cost cutting through PDL usage.

Medical care is often referred to as more of an art than a science. This phraseology indicates that medicine is very individualized and, in certain classes of medication, no two patients may react or respond the same. This phenomenon is known as heterogeneity of response. Soumerai (2004) has suggested that, based on his extensive research, medications that are safer to restrict via a formulary such as a PDL are those that have low heterogeneity. This means that a greater percentage of patients using the medication for the prescribed indication will have the desired response, as seen with $\mathrm{H} 2$ antagonists in the treatment of simple heartburn. Soumerai's implication is that the classes of medication exhibiting high heterogeneity should not be restricted because response rates are inconsistent, even between drugs in the same class. Drugs used to treat certain mental and behavioral illnesses are examples of such medications, including antidepressants, antipsychotics, benzodiazepines, and antiepileptics. Another study attempted to determine the effects of formulary restrictions for selective serotonin reuptake inhibitor (SSRI) antidepressants on utilization of other health care services. In order to determine this point, it had to be assessed whether or not there is interchangeability within the class of SSRIs. Results indicate that switching SSRIs causes an increased incidence of adverse events and discontinuation of treatment, which is the most detrimental effect and can lead to worsening depression, hospitalization, and suicide (Hensley and Nurnberg 2001). This shows the high heterogeneity of response among patients taking SSRIs and the danger associated with imposing restrictions on these drugs.
A number of states have already responded to the growing concern regarding restricting medications, especially among fragile patient populations. In Virginia, the Medicaid PDL program excludes antipsychotics and antiepileptics from being subject to restrictions. They also have two psychiatrists on their pharmacy and therapeutics (P\&T) committee, where drugs are evaluated based on efficacy data to determine whether they will be placed onto the PDL (Finnerty 2003). Other states - such as New York, Kansas, Iowa, and Michigan - have also adopted similar exemptions, with some even broadening the definition to include any medication intended to treat a mental illness. Other classes of medication that certain states prohibit restriction from are HIV/AIDS medication, drugs used for the treatment of cancer, and antirejection drugs for the treatment of organ and tissue transplants (Cauchi et al 2006).

The argument that drug restrictions tend to increase utilization of other areas of healthcare merits serious consideration. As drug restrictions start to affect medications with high heterogeneity of response, we may begin to see an increase in hospital and physician visits. Although many states have not restricted certain drugs associated with sensitive populations, it was discovered that the cost savings after PDL implementation were not as much as desired. This may lead some states to rescind their exclusions in order to save more money, as was attempted in Oregon (Bernasek et al 2004). It is yet to be determined what effect these possible changes would have on spending and utilization in other areas and on the overall health of its beneficiaries.

\section{Criteria used for acceptance of drugs onto a PDL}

A main concern among beneficiaries of Medicaid under new PDL programs has been whether their medications will be covered. State Medicaid departments have assured their beneficiaries that the drugs approved for coverage are the best medications in their respective classes, taking both safety and efficacy into account well before cost considerations. It has been shown, however, that there are many factors involved in the lengthy process of determining the "preferred status" of a medication.

States that have implemented the use of a PDL have all followed the same general courses of action. A P\&T committee, made up of physicians, pharmacists, nurses, and others, is appointed to determine which classes of drugs are to be evaluated for inclusion to the PDL. At this point, pharmacy benefit managers (PBMs) are often contracted to negotiate supplemental rebates (beyond the federally mandated rebates) 
with manufacturers of the drugs in question on behalf of the state. Manufacturers will undoubtedly grant rebates to state Medicaid programs in order to ensure that their drug products will be more closely considered for the state's PDL. Inclusion on such a list would guarantee a generous percentage of the state's population who will be utilizing their drug. Some states have even come together to form coalitions to increase their purchasing power and negotiation leverage with manufacturers. In many cases, those manufacturers who have not negotiated price rebates will not have their drugs considered for inclusion (Texas Health and Human Services Commission 2005).

After rebates are negotiated, the P\&T committees review information acquired from manufacturers, labelers, and other parties. They also consider public testimonies. Safety, efficacy, and clinical outcomes of each considered medication are reviewed publicly. Cost considerations and final recommendations are made behind closed doors due to regulations that allow manufacturer price negotiations to remain private. The P\&T committee then announces its recommendations publicly to be approved by the state Medicaid agency (Texas Health and Human Services Commission 2005). In most states, medications that do not make it onto the PDL are considered "non-preferred"; prior authorization must be obtained for them to be covered by Medicaid.

It is well documented that the P\&T committees and subcommittees entrusted with evaluating medical information do not take their job lightly. Subcommittee reports on single classes of medication are lengthy and filled with clinical trials and other documentation evaluating therapeutic outcomes, adverse events, and safety in numerous patient populations. Nevertheless, there are times that cost considerations come before clinical outcomes in the decision-making process. For example, the P\&T committee of Louisiana announced its recommendations for drugs in a number of therapeutic classes to be included in the state's Medicaid PDL. Two very commonly prescribed medications, Lipitor (for hyperlipidemia) and Norvasc (a calcium channel blocker for hypertension) were noticeably absent from the list. It was stated that the reason for their absence was that Pfizer (New York, NY), the manufacturer of both medications, had not entered into price negotiations with the state (Louisiana Department of Health and Hospitals 2005). Both medications have been placed as non-preferred drugs regardless of evidence of greater therapeutic benefit; For example, reduction of low-density lipoprotein of Lipitor as compared to most other "statins" and evidence of higher overall medical costs associated with switching from Norvasc to another medication in the same class should have been considered (Jones et al 1998; Smith et al 2002). Decisions such as these may result in patients receiving suboptimal treatment in favor of a short-term cost savings. While P\&T committees generally strive to allow for availability of the best-quality medications possible for Medicaid beneficiaries, it seems that cost factors can occasionally win out over safety and efficacy.

\section{Effect of PDL implementation on compliance to new regimens}

Another long-standing issue in the healthcare field is the concern of patient adherence to medication regimens. It is believed that fewer than 50 percent of patients in developed countries who suffer from chronic conditions are adherent to their treatment recommendations (World Health Organization 2003). Noncompliance to therapy and lifestyle modifications in many chronic conditions like hypertension can lead to detrimental effects such as stroke, heart attack, and heart failure.

There are a number of approaches that we have found over the years to minimize the possibility for noncompliance. These methods include patient education on the disease state and importance of treatment, new formulations of drugs that reduce dosing frequency or improve ease of administration, and combining medications that are commonly used together in order to minimize pill burden. Except for patient education, these other methods tend to be newer and more innovative than their predecessors are. They are often more expensive and less likely to be available to Medicaid patients under a restrictive drug formulary.

Drug manufacturers can create extended-release preparations of their drugs in order to decrease dosing frequency and pill burden on a patient; two examples are Toprol XL, the extended-release version of metoprolol for hypertension, and Effexor XR, an extended-release version of Effexor indicated for treatment of depression and anxiety. While these formulations help to increase patient compliance, they also cost more due to new patents for the brand. In Georgia, for example, generic metoprolol is a preferred drug on the state's PDL, but Toprol XL is not covered (Georgia Medicaid/PeachCare Preferred Drug List 2006). Similarly, in Florida, regular-release Effexor is the favored drug on the PDL over Effexor XR (Florida Medicaid Preferred Drug List 2006). One study conducted on diabetic patients attempted to determine the difference in compliance between patients taking a once daily versus a twice-daily oral antidiabetic drug regimen. The study showed increased compliance and improved glycemic control in the once-daily patient group. 
While the study could not specifically attribute the glycemic control to either adherence or therapeutic superiority, there is still a clear correlation between dosing frequency and general pill burden and therapy adherence (Kardas 2005). If states start to utilize therapies that are simpler for patients to use, there is a good chance they could avoid incurring payments in other areas of healthcare that would be due to poor compliance.

\section{Potential effects of medication restriction on quality of care}

To analyze the potential effects that PDLs and other medication restrictions may have on quality of patient care, we must first define good quality of care. Aside from the personal responsibilities we have toward our own healthcare, physicians have the ability to evaluate our health status individually and determine what means of therapy is best, whether it be by lifestyle modifications or by medication intervention. The problem comes when external factors influence a physician's judgment toward utilizing therapies that may not be best for a particular person. Thus, good quality of care may be described when patients receive the type(s) of therapy most appropriate to treat their condition.

With more restrictive policies in place for drug approval, physicians are more likely to use a different therapy than what may be optimal in order to avoid the hassles of receiving authorization. In Illinois, after PDL implementation, the percentage of shares of off-PDL prescriptions dropped from 13.5 percent to 1.6 percent. In Louisiana, where the approval process is much less stringent, there was less of a reduction in prescribing for non-preferred drugs (Virabhak and Shinogle 2005). While this is indicative of the utility of PDLs and strict PA processes in reducing spending on non-preferred drugs, we must also consider the chance that perhaps the quality of therapy was not optimal, because other therapies were utilized prior to PDL implementation.

Effects of Medicaid PDL implementation may even be seen in the general population due to a phenomenon called the "spillover effect." This is when a physician's prescribing behavior is altered, not only for Medicaid patients, but also for patients who are not subject to the same formulary restrictions. In Illinois, it was observed that among physicians who mainly saw Medicaid patients, who were subject to formulary restrictions, there was a significant drop in the writing of non-preferred drugs for those patients who had other third party coverage (Virabhak and Shinogle 2005). Some third parties adjust their own formularies to mimic that of the state Medicaid formulary. Unfortunately, such "bandwagon effects" were unexplainable. This example indicates the high level of influence that PDLs can have on some physicians, which may lead to spillover effects on the general population.

Another study determined that physicians who saw patients associated with PacifiCare, a large third-party provider in Cypress, California, tended to prescribe the same drugs for non-PacifiCare patients as were on the PacifiCare formulary. The observed spillover effects indicate that greater restrictions have a tendency to influence a physician's prescribing patterns universally (Wang and Pauly 2005). There are some instances where switching medications within a class had no significant effect on therapeutic outcome. In the cases where there is a difference between drugs, however, there is the possibility for compromised quality of care when barriers are in place to dissuade the use of those drugs. Whether or not the changes in prescribing patterns lead to patients not receiving optimal and personalized treatment, it is an issue of great concern that must still be studied.

\section{Administrative costs associated with PDLs}

There have been questions raised as to whether implementation of a PDL program for Medicaid will save money because of decreased drug spending or if it will cost more money because of the administrative burden of processing authorizations. Considering the drug rebates that states obtain and the effect of formulary restrictions on physician prescribing patterns, it is no surprise that states like Iowa have found significant cost savings associated with PDL implementation. The Iowa state Medicaid advisory council reported an average savings of $\$ 17.8$ million for 2005 , and estimates a savings of \$22 million in 2006 (State of Iowa Department of Human Services 2006). The analysis of cost savings neglected to mention any administrative costs of the program.

A number of areas must be considered when reviewing costs associated with PDL implementation. Firstly, there are costs associated with forming and executing P\&T committee meetings; these costs are salaries that are figured into the state's budget. Secondly, there are the costs of contracting PBMs to negotiate rebates with drug manufacturers. Lastly, Medicaid must also contract with a PA service that will receive authorizations requests, evaluate them, hand them off to physicians or pharmacists hired to evaluate these more complex requests, and either approve or deny a claim. The Texas Medicaid PDL Annual Report stated that their administrative cost for contracting both PBM and PA provider services was about \$3.3 million from November 2003 
to August 2004. It is now estimated that the PDL program will maintain a drug savings of about $\$ 140$ million for the 2004-2005 biennium (Texas Health and Human Services Commission 2005). This shows that the PDL implementation in Texas will bring much more savings in drug spending than the cost to administer the program.

State Medicaid agencies have not accounted for the administrative costs for other healthcare providers. Both physicians' offices and pharmacies have noted increases in time spent processing authorizations; time that could be better spent interacting with patients and reducing medication errors. A survey was conducted in Maine that indicated that 98 percent of providers reported an increase in telephone traffic after implementation of the PA program (MaineCare Advisory Committee 2005). This, in turn, has resulted in an increase in administrative overhead costs and provider time constraints. One physician said, "It takes too much time to fill out forms and that time could be spent returning patient calls and reaching patients." Similarly, another provider indicated, "[the PA process] requires additional staff to meet the paper work needs. Ten to fifteen hours weekly are spent filing PA's, on the phone with GHS staff, and combing through charts for the increasing amount of information demanded to get PA's approved." While the direct administrative costs do not seem to outweigh the savings associated with decreased drug spending, the effect on administration in other areas of healthcare should also be considered.

\section{Patient and provider satisfaction with PDL programs}

There has been much controversy surrounding the implementation of PDLs in states throughout the country. Many healthcare providers and patient advocates are concerned that drug preference will go to the lowest bidding manufacturer as opposed to the most effective and safest medication. In New York State, the proposed PDL bill did not even pass legislation until there was a safeguard for all medications prescribed for mental illness, HIV/AIDS, cancer, and organ and tissue transplantation. While different states are implementing different policies, most will still be utilizing the PA method to deter usage of higher-cost medications. Many healthcare providers have issues with mandatory PAs and are becoming frustrated. One provider in Maine remarked, "Our practice is considering limiting the number of Medicaid patients; other surrounding practices have done so and [their patients] are now flooding into our office" (MaineCare Advisory Committee 2005). Such decisions to limit Medicaid patients would greatly decrease the access such patients would have to healthcare and may deter them from seeking preventative care. This may cause them to end up in an emergency room and incur greater expenses. Despite the push for preventative healthcare today, it seems the PDL's effects on providers may result in less preventative care and more chronic medical issues, which are more costly in the long run.

In addition, the conflict between quality and costs is inevitable when decisions must be made between clinical and management goals, it disrupts the doctor-patient relationship. Patients believe more and more that physicians no longer have a duty to deliver quality care. With concepts of salary bonuses for those who provide the most inexpensive care and cuts for those who provide costly care, there is a conflict of interest that the patients can sense (Avorn 2002). PDLs and formularies continue to bring such conflicts into the examination room and push patients further and further from their physicians. This is not to say physicians should not be cost conscious; on the contrary, physicians have a responsibility to be aware of the economic consequences of their clinical decisions, but during this process, the insurers, employers, payers, and others should not push them in numerous directions.

There are known impacts of formulary restrictions on patient satisfaction across many third-party payers. For MCOs, this can be a large problem; their business is dependent on providing insurance to patients, without patients, there is no business. Even in the arena of PA for referrals and medical procedures, many MCOs found themselves dropping PAs due to the high administrative costs and low patient satisfaction. For example, UnitedHealth dropped its requirements after finding that they denied fewer than 2 percent of treatment requests (Curtiss 2002). Within the next year, the organization had saved $\$ 110$ million in administrative costs and experienced a 26 percent decrease in member complaints as well as a 21 percent growth in membership the following year. Blue Cross of California experienced similar benefits after dropping their PA program. Though loss of members is not an issue in the Medicaid program, they should still be concerned with patient satisfaction as well as decreasing administrative costs.

In Maine, there have been numerous cases of patient dissatisfaction since the implementation of a PA program for prescription drugs for the elderly in 2001 (MaineCare Advisory Committee 2005). For example, one patient suffered from multiple chronic conditions, including severe osteoarthritis, hypothyroidism, chronic 
renal failure, chronic lower back pain, type II diabetes, hypertension, severe dementia, and depression. She began using OxyContin ${ }^{\circledR}$ (Purdue Pharma L.P., Stamford, CT) for pain management in 1999. Her treatment was discontinued. When her doctor submitted a request for PA for OxyContin ${ }^{\circledR}$ in March 2004, the Department of Health and Human Services for the MaineCare Program denied it, claiming that the patient had to try as many as nine different pain medications before the PA for OxyContin ${ }^{\circledR}$ could be approved. An appeal of the denial of the PA was made on behalf of the patient, but she passed away before a decision was made. Another patient suffering from attention deficit/ hyperactivity disorder was denied coverage of Provigil ${ }^{\circledR}$ (Cephalon, Frazer, PA) because of the PA program. Even one year after the PA was requested, the case was still in the appeal process. Each state's Medicaid PDL program varies slightly with regard to certain requirements before approval can be made for a restricted drug. Some requirements are much more lenient, such that there needs only to be documentation of previous success on the medication or previous failure of the preferred drug. In either situation, the hurdles involved in obtaining a desired "non-preferred" medication represent an annoyance at best and a health risk at worst to the patient.

\section{Recommendations for cost containment}

In consideration of all these facts, one assumes that there must be a large advantage, as far as cost reduction, in implementing PDLs. However, prescription drugs comprised only 11 percent of the Medicaid budget in 1999. A greater percentage of the expenditures were allocated toward institutional long-term care, and inpatient and outpatient hospital visits (29 percent and 19 percent, respectively) (Rudolph and Lubitz 1999). Figure 2 shows approximately how much of each dollar was being spent in each segment of healthcare for Medicaid in 2002; this illustrates the small part that drug spending has played in the Medicaid budget. A study conducted by Strunk et al (2002) commented that in 2001, despite the 12 percent increase in healthcare spending per capita, this increase reflected a greater use of hospital services as well as an increase in hospital payment rates. In fact, in 2001 - as was seen in 2000 - prescription drug spending growth declined. Therefore, an economic evaluation seems

\section{Nation's Health Dollar, Where It Went: CY 2002}

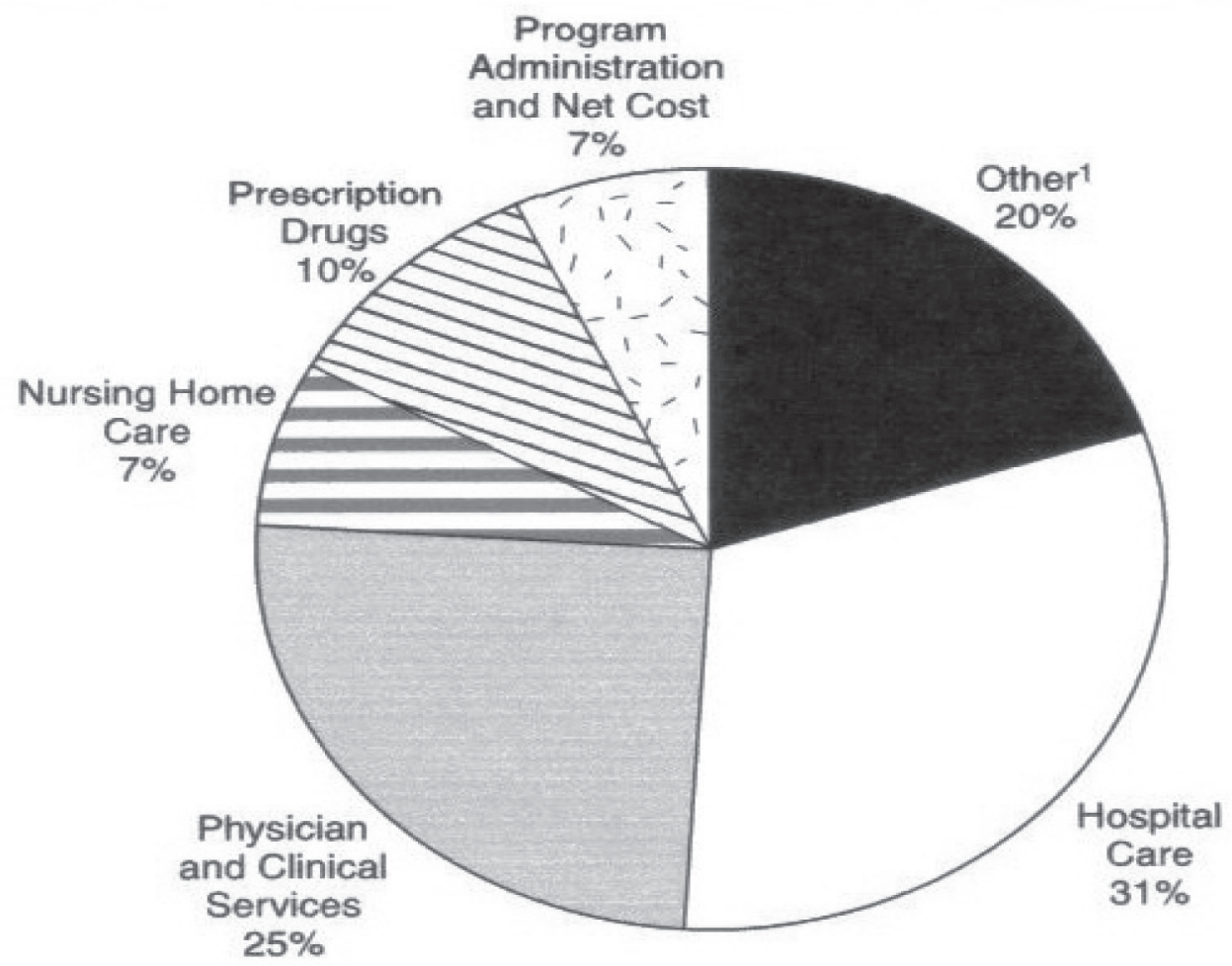

Figure 2 Illustration of the approximate cost of each segment in the health field expressed as a percentage of the nation's health dollar (Health Care Financing Administration 2004). 'Includes dental services, other professional services, home health, durable medical products, over-the-counter medicines and sundries, public health, research and construction. 
necessary to judge whether cost-cutting tactics in the area of pharmaceuticals, such as PA, are the most beneficial.

It seems that the approach of merely limiting prescription drugs in order to alleviate a budget deficit in Medicaid is illogical. In contrast, every component of the healthcare industry should be reviewed as part of reducing healthcare costs. Though a decrease in pharmaceutical costs may seem like a great place for cost containment due to the ability to see direct changes in drug sales, medications are the most cost-effective means of treating chronic illnesses. Therefore, use of the healthcare budget toward pharmaceuticals may be more effective than using that money in other areas such as hospital stays.

Medications and lifestyle modifications are the best forms of prevention for cardiac diseases such as stroke and heart attack. Figure 3 illustrates the cumulative cost of strokes in this country. The economic impact of such diseases is tremendous, with the bulk of the cost being associated with hospital stays, nursing home placement, and the indirect cost of morbidity and mortality. These segments alone comprise about 89 percent of the cost of the disease. Even if spending for drug treatment were to increase, the subsequent reduction in other segments would be well worth the cost, both economically and individually.

To further illustrate that prescription drugs are one of the most effective and least expensive segment in healthcare, Lichtenberg (2005), as mentioned in Bussing-Burks (2006), did a study that indicated that replacing 1000 old prescription drugs with 1000 new prescription drugs resulted in drug costs increasing by $\$ 18,000$ with hospital costs decreasing by $\$ 44,469$. Lichtenberg's data was obtained via the 1996 Medical Expenditure Panel Survey. This study provides data that confirms that drug age influences effectiveness, resulting in changes in medical expenses.

With most states in the country implementing PDLs or some other form of drug spending containment in their Medicaid programs, there must be a balance maintained

\section{Estimated Cost of Stroke in the US}

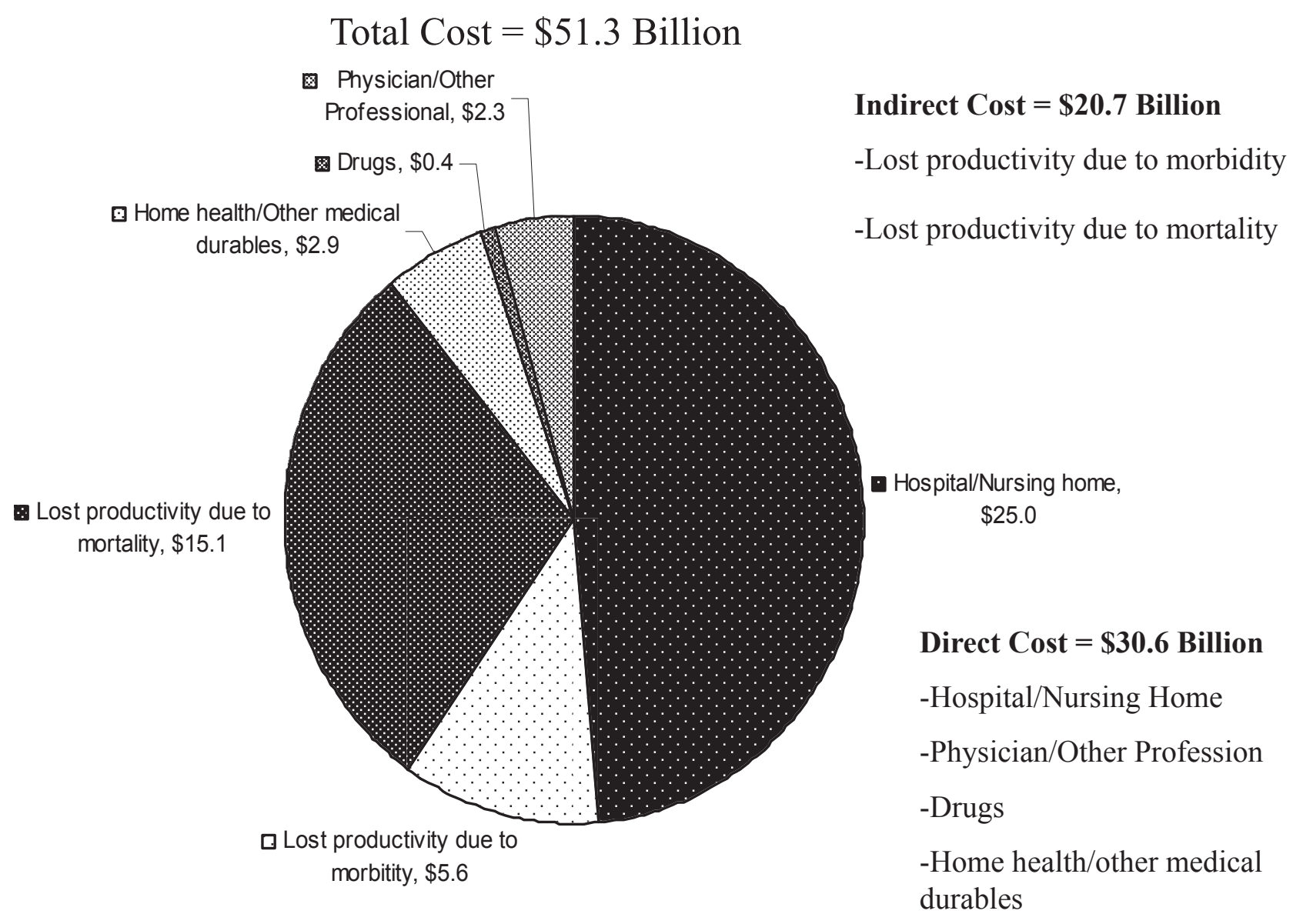

Figure 3 The estimated cost of stroke in the United States (AHA 2000). 
between cost savings and providing good quality and timely medical care. This has proven to be a difficult task, with some states being forced to abandon their desires to have an unrestricted formulary due to critical budget crises. New York State, for example, has consistently offered many optional programs such as psychiatric services, podiatry services, and many more to Medicaid beneficiaries. In turn, New York spends more money on Medicaid services than the states of California and Texas combined (Center for Governmental Research, Inc. 2004). Oregon has implemented a Practitioner-Managed Prescription Drug Plan (PMPDP) where the PDL is only a guide as to which drugs strike the best balance between therapeutic benefit and cost-effectiveness; its usage is entirely voluntary (Bernasek et al 2004). While this is an excellent way to ensure that practitioners retain autonomy in treating their patients, it has not proven to have the best impact on cost containment.

It appears that the ideal PDL program would be one that has identified the most cost-efficient therapies in classes of medication with therapeutic responses that are practically universal and repercussions minimal. In sensitive conditions and in those classes where there is high heterogeneity of response, cost-efficient medications should be highlighted, for informational purposes, but not mandated. Physicians should be able to make the best decisions for each individual patient, and be able to switch medications if necessary by simply stating so on the prescription as opposed to performing an arduous and time-consuming PA request that leaves the physician, the pharmacy, and the patient frustrated. One example of such a policy is seen in the Medicaid program of Alaska. Preferred drugs and generics are available without authorization. Other "non-preferred" drugs require only a statement of medical necessity on the prescription in order to be covered, similar to a dispense as written (DAW) designation (Preferred Drug List Program 2006). Voluntary lists encourage the use of cost-effective medications but do not dissuade practitioners from utilizing any therapy that may be medically necessary. At this time, there has been no statement released on the state's costs and savings after program implementation.

Healthcare cannot be managed in a short-term mindset, and decisions should not be made without looking at their reverberations in the future. Nevertheless, our actions within the healthcare industry have repeatedly shown that we focus on short-term benefits in many of our decisions. Examples of such concern for the short-term costs are rampant in healthcare because there may be one component responsible for a large portion of the bill. However, in the end, some "quick-fix" cost-saving policies may not always be the most beneficial ones, economically. We are beginning to see the effects in the Medicare Part D drug plan, where seniors who have found themselves in a "doughnut hole" are now paying full price for their medications. This has shown to decrease compliance and usage of medication, which can clearly be detrimental, especially in patients with chronic diseases (Stuart et al 2005). It remains to be seen how the PDL programs implemented around the country will affect the Medicaid population.

\section{Conclusion}

The studies and literature we have examined illustrate that there are a number of issues to be considered concerning the implementation of a PDL in a state Medicaid program. Some key issues include the effects on use of other healthcare services, the criteria used by P\&T committees for acceptance of drugs onto a PDL, the effect of switching medication therapy on patient compliance, the possible effects of drug restriction on quality of care, the administrative costs associated with the program, and the impact such reforms may have on physician and patient satisfaction. Reducing drug spending via PDLs and drug authorizations is a common practice in most states, and is an effective way to save money. The best way to approach reforms in Medicaid policies, however, is to consider the effects that such programs will have on the future of healthcare and reinforce the importance of proper prescription drug use in improving health outcomes. Issues concerning conflicts between cost savings and quality of care illustrate the need for more studies on cost expenditures of open and closed formularies that also analyze all aspects of the subsequent quality of care received.

\section{Acknowledgment}

The authors wish to thank Pearl Weisinger and Christine Meck for help in editing this article.

\section{References}

Avorn J. 2002. Balancing the cost and value of medications. Pharmacoeconomics, 20:67-72.

[AHA] American Heart Association. 2000. Heart and stroke statistical update. Dallas, TX: American Heart Association.

Bernasek C, Mendelson D, Padrez R, et al. 2004. Oregon's Medicaid PDL: Will an evidence-based formulary with voluntary compliance set a precedent for Medicaid?

Bussing-Burks M. 2006. Benefits of newer prescription drugs exceed their costs [online]. Accessed 10 January 2006. URL: http://www.nber. org/digest/oct01/w8147.html

Carroll NV. 2002. How effectively do managed care organizations influence prescribing and dispensing decisions? Am J Manag Care, 8:1041-54. 
Cauchi R, Folkemer D. 2006. Recent Medicaid prescription drug laws and strategies, 2001-2005 [online]. Accessed 1 September 2006. URL: http://www.ncsl.org/programs/health/medicaidrx.htm

Center for Governmental Research, Inc. 2004. On the road to Medicaid reform: a beginning [online]. Accessed 28 August 2006. URL: http:// www.cgr.org/ICSG/Articles/?id=155

Curtiss FR. 2002. Managing care via prior authorization (PA) programs? Manag Care Pharm, 8:296.

Curtiss FR. 2003. Prior authorization to manage drug utilization and costs. Manag Care Pharm, 9:95.

Finnerty PW. 2003. Status report on Virginia's Medicaid Preferred Drug List (PDL) Program. Joint Commission on Health Care Behavioral Health Subcommittee; October 7 [online]. Accessed 29 August 2006. URL:http://www.dmas.virginia.gov/downloads/pdfs/pharmpdl_status_report_10-07-03.pdf

Florida Medicaid Preferred Drug List Updated 20 July 2006 [online]. Accessed 1 September 2006. URL: http://www.fdhc.state.fl.us/ Medicaid/Prescribed_Drug/pharm_thera/fmpdl.pdf

Gencarelli DM. 2003. Medicaid prescription drug coverage: state efforts to control costs. National Health Policy Forum Issue Brief, May 10: No. 790.

Georgia Medicaid/PeachCare Preferred Drug List revised 25 January 2006 [online]. Accessed 1 September 2006. URL: http://dch.georgia.gov/vgn/ images/portal/cit_1210/5/31/48919362GME_PDL_alpha_02-06.pdf

Gleason PP, Gunderson BW, Gericke KR. 2005. Are incentive-based formularies inversely associated with drug utilization in managed care? Ann Pharmacother, 39:339-45.

Hamel MB, Epstein AM. 2004. Prior-authorization programs for controlling drug spending. $N$ Engl J Med, 351:2156-8.

Health Care Financing Administration. 2004. Office of the Actuary National Health Statistics Group.

Hensley PL, Nurnberg HG. 2001. Formulary restriction of selective serotonin reuptake inhibitors for depression. Pharmacoeconomics, 19:973-82.

Huskamp HA, Deverka PA, Epstein AM, et al. 2003. The effect of incentive-based formularies on prescription-drug utilization and spending. N Engl J Med, 349:2224-32.

Jones P, Kafonek S, Laurora I, et al. 1998. Comparative dose efficacy study of atorvastatin versus simvastatin, pravastatin, lovastatin, and fluvastatin in patients with hypercholesterolemia (the CURVES study). Am J Cardiol, 81:582-7.

Kaiser Commission on Medicaid and the Uninsured [online]. Accessed 29 August 2006. URL: http://www.kff.org/medicaid/upload/Oregon-sMedicaid-PDL-Will-an-Evidence-Based-Formulary-with-VoluntaryCompliance-Set-a-Precedent-for-Medicaid.pdf

Kardas P. 2005. The DIACOM study (effect of DosIng frequency of oral antidiabetic agents on the COMpliance and biochemical control of type 2 diabetes). Diabetes Obes Metab, 7:722-8.

Lichtenberg FR. 2005. The effect of access restrictions on the vintage of drugs used by Medicaid enrollees. Am J Manag Care, 11:SP7-13.

Louisiana Department of Health and Hospitals. 2005. Medicaid Pharmaceutical and Therapeutics Committee Meeting Minutes, March 9, 2005 [online]. Accessed 1 September 2006. URL: http://www.dhh.louisiana. gov/offices/publications/pubs-134/Approved\%20BY\%20P\&TMin03 \%5B1\%5D.09.05.pdf
MaineCare Advisory Committee. 2005. The MaineCare Advisory Committee's prior authorization subcommittee report and recommendations on prior authorization for prescription drugs in the Maine Care and Drugs for the Elderly Programs. January 19.

Martinez G. 2003. Assemblyman Peter M. Rivera questions Senate Medicaid report, calls recommendations a blue print for treating New York's poor like guinea pigs [online]. Accessed 28 August 2006. URL: http://www. somoselfuturo.com/news_med2.htm

Mattera MD. 2004. A way to curb healthcare costs? Med Economics, 3:8.

Murawski MM, Abdelgawad T. 2005. Exploration of the impact of preferred drug lists on hospital and physician visits and the costs to Medicaid. Am J Manag Care, 11:SP35-42.

Preferred Drug List Program. 2006. Juneau (AK). Health and Social Services, State of Alaska [online]. Updated 8 August 2006. Accessed 1 September 2006. URL: http://www.hss.state.ak.us/dhcs/PDL/default.htm

Rudolph NV, Lubitz J. 1999. Capitated payment approaches for Medicaidfinanced long-term care services. Health Care Financ Rev, 21:51-64.

Seay JD. 2004. NAMI-NYS Action Agenda for 2004 [online]. Accessed 28 August 2006. URL: http://www.naminys.org/action_04.htm

Silow-Carroll S, Altera T. 2004. Stretching state health care dollars: pooled and evidence-based pharmaceutical purchasing. The Commonwealth Fund, October, $\mathrm{p} 2$.

Smith GM, Harper IM, Stowers AD. 2002. A cost analysis of switching calcium channel blockers: a one-year post-formulary decision review. Pharmacy Therapeutics, 27:333-44.

Soumerai SB. 2004. Benefits and risks of increasing restrictions on access to costly drugs in Medicaid. Health Affairs, 23:135-46.

State of Iowa Department of Human Services. Medical Assistance Advisory Council Summary of Meeting, February 1, 2006 [online]. Accessed 30 August 2006. URL: http://www.dhs.state.ia.us/dhs2005/ime/docs/ 2006FebMinutesNew.doc

Strunk BC, Ginsburg PB, Gabel JR. 2002. Tracking health care costs: growth accelerates again in 2001. Health Affairs, Jul-Dec; Suppl Web Exclusives:W299-310.

Stuart B, Simoni-Wastila L, Chauncey D. 2005. Assessing the impact of coverage gaps in the Medicare Part D drug benefit. Health affairs [exclusively on the internet]. Accessed 28 August 2006. URL: http://www.allhealth.org/BriefingMaterials/HealthAffairsAssessingTheImpact-46.pdf

Texas Health and Human Services Commission. 2005. Preferred Drug List annual report: January 2005 [online]. Accessed 25 August 2006. URL: http://www.hhsc.state.tx.us/HCF/vdp/reports/010105_PDLAR.pdf

Virabhak S, Shinogle JA. 2005. Physicians' prescribing responses to a restricted formulary: the impact of Medicaid preferred drug lists in Illinois and Louisiana. Am J Manag Care, 11:SP14-20.

Wang RY, Pauly MV. 2005. Spillover effects of restrictive drug formularies: a case study of PacifiCare in California. Am J Manag Care, 11:24-6.

World Health Organization. 2003. Adherence to long-term therapies: evidence for action [online]. Accessed 27 August 2006. URL: http://www. who.int/chronic_conditions/adherencereport/en/ 
